\title{
Dynamic linear mixed models with ARMA covariance matrix
}

\author{
Eun-Jeong $\operatorname{Han}^{a}$, Keunbaik Lee ${ }^{1, a}$ \\ ${ }^{a}$ Department of Statistics, Sungkyunkwan University, Korea
}

\begin{abstract}
Longitudinal studies repeatedly measure outcomes over time. Therefore, repeated measurements are serially correlated from same subject (within-subject variation) and there is also variation between subjects (betweensubject variation). The serial correlation and the between-subject variation must be taken into account to make proper inference on covariate effects (Diggle et al., 2002). However, estimation of the covariance matrix is challenging because of many parameters and positive definiteness of the matrix. To overcome these limitations, we propose autoregressive moving average Cholesky decomposition (ARMACD) for the linear mixed models. The ARMACD allows a class of flexible, nonstationary, and heteroscedastic models that exploits the structure allowed by combining the AR and MA modeling of the random effects covariance matrix. We analyze a real dataset to illustrate our proposed methods.
\end{abstract}

Keywords: Cholesky decomposition, within-subject variation, longitudinal data, heteroscedastic, covariance matrix, positive definite, serial correlation

\section{Introduction}

Longitudinal studies repeatedly measure outcomes over time. Therefore, repeated measurements are serially correlated from same subject (within-subject variation) and there is also variation between subjects (between-subject variation). The serial correlation and the between-subject variation must be taken into account to make proper inference on covariate effects (Diggle et al., 2002). For this reason linear mixed models (LMMs) have been widely used to analyze longitudinal data. In the LMMs, there are conditional covariance matrix and random effects variance to explain the within-subject variation and the between-subject variation, respectively (Laird and Ware, 1982). However, the estimation of the conditional covariance matrix is challenging because its estimate needs to be positive definite and high-dimensional. In addition, the covariance matrix can be heteroscedastic depending on covariates. In this paper, we focus on modeling of the conditional covariance matrix in the LMMs.

Two Cholesky decompositions were recently proposed to model the covariance matrix: modified Cholesky decomposition (MCD) and moving average Cholesky decomposition (MACD). The MCD factors the inverse covariance matrix into generalized autoregressive parameters (GARPs) and innovation variances (IVs) (Pourahmadi, 1999, 2000). Pan and MacKenzie (2003, 2006) extended the MCD to deal with unbalanced longitudinal data and to explain heterogeneity in the covariance matrix, respectively. The MCD approach was also used for the random effects covariance matrix in linear mixed models (Daniels and Zhao, 2003; Pan and MacKenzie, 2007) and generalized LMMs (Kim et

\footnotetext{
${ }^{1}$ Corresponding author: Department of Statistics, Sungkyunkwan University, 25-2, Sungkyunkwan-ro, Jongno-gu, Seoul 03063, Korea. E-mail: keunbaik@skku.edu

Published 30 November 2016 / journal homepage: http://csam.or.kr

(C) 2016 The Korean Statistical Society, and Korean International Statistical Society. All rights reserved.
} 
al., 2016; Lee, 2013; Lee et al., 2012; Lee and Sung, 2014), respectively. In the MACD, the (random effects) covariance matrix was factored into generalized moving average parameters (GMAPs) and IVs instead of the inverse covariance matrix (Lee and Yoo, 2014; Zhang and Leng, 2012). Kim et al. (2016) exploited the MACD to model random effects covariance matrix in cumulative logit random effects models.

Both decompositions remove the positive definiteness restriction of the (random effects) covariance matrix using a log-linear model for IVs. In addition, using the log-linear model for IVs and the regression model for GARPs and GMAPs, the number of parameters for the (random effects) covariance matrix is reduced and the covariance matrix allows heterogeneity.

Lee et al. (2016) recently proposed linear models with a heterogeneous autoregressive moving average (ARMA) marginal covariance matrix using ARMA Cholesky decomposition (ARMACD). The decomposition combines the MCD and MACD to create a more flexible decomposition of the marginal covariance matrix and captures a high-order AR structure of the covariance matrix. The decomposition also provides simple conditions for positive definiteness in terms of IVs. The decomposition also allows flexible nonstationary models that can be more parsimonious than models using either the MCD or MACD alone. In this paper, we propose a Bayesian modeling of linear mixed model that allows a modeling of the conditional covariance matrix, not just a marginal covariance matrix. Our proposed model is similar to Pan and MacKenzie's (2007) model, but with a heterogenous ARMA covariance matrix using ARMACD.

This paper is organized as follows. In Section 2, we propose modeling of the random effects covariance matrix using the ARMACD and present Bayesian inference using a Gibbs sampler with random walk Metropolis-Hastings steps. In Section 3, we apply our proposed models to data from a recent lung cancer study. Finally, we summarize and propose future work in Section 4.

\section{ARMA Cholesky factor models for conditional covariance matrix}

We propose a new class of models of the conditional covariance matrix for longitudinal data that relies on the parameters in the new ARMACD.

\subsection{Proposed model}

Let $y_{i}=\left(y_{i 1}, \ldots, y_{i n_{i}}\right)^{T}$ be the longitudinal response vector of the $i$ th subject $(i=1, \ldots, N) ; y_{i j}$ is the response at time $t_{i j}$ for subject $i$ where $t_{i j}$ is discrete time $t_{i j}=1,2, \ldots, T$. Here, $t_{i j}$ could be months, weeks, days or hours (in our example in Section 3, it is 'months'). The constant $T$ is determined by the potential maximum follow-up time where a longitudinal measurement can be taken in the study. We assume that the responses for different subjects are independent. Let $x_{i j}$ indicate covariates corresponding to $y_{i j}$. We assume the $y_{i j}$ follows the LMM which is given by

$$
\begin{aligned}
y_{i j}-x_{i j}^{T} \beta & =\sum_{l=1}^{j-1} \phi_{i j l}\left(y_{i j-l}-x_{i j-l}^{T} \beta\right)+c_{i j}^{T} b_{i}+\sum_{k=1}^{j-1} l_{i j k} \epsilon_{i j-k}+\epsilon_{i j}, \\
b_{i} & \sim N\left(0, G_{b}\right),
\end{aligned}
$$

where $\beta$ is a $p \times 1$ coefficients vector of $x_{i j}, b_{i}=\left(b_{i 1}, \ldots, b_{i q}\right)^{T}$ is a $q \times 1$ dimensional vector of random effects, $c_{i j}$ is a $q \times 1$ subset of $x_{i j}, G_{b}$ is the random effects covariance matrix, and $\epsilon_{i j} \stackrel{\text { indep. }}{\sim} N\left(0, \sigma_{i j}^{2}\right)$. Note that $b_{i}$ is a $q \times 1$ subject-specific random effects and $G_{b}$ is a general $q \times q$ covariance matrix. 
We can rewrite (2.1) in matrix form as

$$
T_{i}\left(y_{i}-X_{i} \beta\right)=C_{i} b_{i}+L_{i} \epsilon_{i}
$$

where $y_{i}=\left(y_{1}, \ldots, y_{n_{i}}\right)^{T}, X_{i}=\left(x_{i 1}, \ldots, x_{i n_{i}}\right)^{T}, T_{i}$ is a unit lower triangular matrix having ones on its diagonal and $-\phi_{i, j l}$ at its $(j, l)^{\text {th }}$ position for $j>l$, and $L_{i}$ is a unique lower triangular matrix having ones on its diagonal and $l_{i, j k}$ at its $(j, k)^{t h}$ position for $j>k, X_{i}=\left(x_{i 1}, \ldots, x_{i n_{i}}\right)^{T}$ is the design matrix, and $C_{i}=\left(c_{i 1}, \ldots, c_{i n_{i}}\right)^{T}$. In this paper, we assume that $X_{i}$ is of full rank. From (2.3), we have

$$
T_{i} \Sigma_{i} T_{i}^{T}=C_{i} G_{b} C_{i}^{T}+L_{i} D_{i} L_{i}^{T}
$$

where $\Sigma_{i}$ is the $n_{i}$-dimensional covariance matrix for $y_{i}$ and $D_{i}$ is diagonal with $\sigma_{i j}^{2}=\operatorname{var}\left(\epsilon_{i j}\right)$ as its diagonal entries. The GARPs and the GMAPs are respectively represented by $\phi$ and $l$, and $\sigma^{2}$ denotes the IVs.

Proposition 1. $\Sigma_{i}$ in (2.4) is positive definite if both $G_{b}$ is positive definite and the diagonal elements of $D_{i}$ are all positive.

Proof: : Let $x$ be an arbitrary nonzero vector. Since $\Sigma_{i}=T_{i}^{-1}\left(C_{i} G_{b} C_{i}^{T}+L_{i} D_{i} L_{i}^{T}\right) T_{i}^{-T}, x^{T} \Sigma_{i} x=$ $x T_{i}^{-1}\left(C_{i} G_{b} C_{i}^{T}+L_{i} D_{i} L_{i}^{T}\right) T_{i}^{-T} x$. For $z_{1}=C_{i}^{T} T_{i}^{-T} x$ and $z_{2}=L_{i}^{T} T_{i}^{-T} x, x^{T} \Sigma_{i} x=z_{1}^{T} G_{b} z_{1}+z_{2}^{T} D_{i} z_{2}$. Since $G_{b}$ is positive definite and $\sigma_{i j}^{2}>0$ for $j=1, \ldots, n_{i}, x^{T} \Sigma_{i} x>0$.

The GARPs, GMAPs, and IVs can be modeled using time and/or subject-specific covariate vectors $w_{i, j l}, z_{i, j k}$, and $h_{i, j}$ by setting

$$
\begin{aligned}
\phi_{i, j l} & =w_{i, j l}^{T} \alpha, \quad l_{i, j k}=z_{i, j k}^{T} \gamma, \\
\log \left(\sigma_{i, j}^{2}\right) & =h_{i, j}^{T} \lambda,
\end{aligned}
$$

where $\alpha, \gamma$, and $\lambda$ are $a \times 1, b \times 1$, and $c \times 1$ vectors of unknown parameters, respectively. Note that the loglinear model for $\sigma_{i j}^{2}$ is assumed to satisfy the positive definiteness of $\Sigma_{i}$ by theorem. We also note that we jointly model the mean and covariance structures of responses in terms of generalized linear models from (2.1) and (2.5).

In models (2.5), $w_{i, j l}, z_{i, j l}$, and $h_{i, j}$ are the design vectors to model the GARPs, GMAPs, and IVs, respectively. These design vectors can include subject-specific covariates for the heteroscedastic covariance matrix. For example, time lag, $\left|t_{i j}-t_{i l}\right|$ in the design vectors $w_{i, j l}$ and/or $z_{i, j l}$ specifies higher lag models. $t_{i j}$ in the design vector $h_{i, j}$ specifies the linearity in $t_{i j}$. Table 1 in Section 3 provides more examples. Overall, the covariance matrix with these models (2.5) includes heteroscedastic and nonstationary processes for $Y_{i j}$.

There are several advantages in ARMACD modeling. First, the parameters in the ARMACD allow flexibility via more parsimonious models compared to higher order AR or MA models (Lee et al., 2016). The MCD and MACD are special cases of the ARMACD which provides a unified framework to model the random effects covariance matrix of linear mixed models. Second, the ARMA modeling provides better forecasting performance than competing higher order AR or MA models (Hansen and Lunde, 2005). Third, we can construct more complex (but still parsimonious) 'ARMA' types models that allow nonstationarity and heterogeneity as described above. 


\subsection{Bayesian approach}

We derive the likelihood function for the model specified in Subsection 2.1. The parameters in model (2.5) are the regression coefficients which ranges on $(-\infty, \infty)$. Thus, normal priors are commonly used for the parameters and guarantee the propriety of posterior distributions. In the absence of any prior information, the normal priors with large prior variances remains relatively objective. The prior distribution for $G_{b}$ is the inverse Wishart distribution that is a conjugate prior. The priors distributions for the model with the ARMA structure of random effects covariance matrix are given by

$$
\begin{aligned}
\beta & \sim N\left(0, \sigma_{\beta}^{2} I\right), \\
\alpha & \sim N\left(0, \sigma_{\alpha}^{2} I\right), \\
\gamma & \sim N\left(0, \sigma_{\gamma}^{2} I\right), \\
\lambda & \sim N\left(0, \sigma_{\lambda}^{2} I\right), \\
G_{b} & \sim \operatorname{InvWishart}\left(G_{0}^{-1}, v\right),
\end{aligned}
$$

where InvWishart is an inverse Wishart prior, parameterized to have mean $(v-q-1)^{-1} G_{0}$. In general, $\sigma_{\beta}^{2}, \sigma_{\alpha}^{2}, \sigma_{\gamma}^{2}$, and $\sigma_{\lambda}^{2}$ are in general large to be noninformative (Daniels and Zhao, 2003), and $v$ is small number.

Let $\theta=\left(\beta^{T}, \alpha^{T}, \gamma^{T}, \lambda^{T}, G_{b}\right)$ and let $\theta_{-\delta}$ be $\theta$ excluding $\delta$. The joint distribution is given by

$$
\begin{aligned}
& p(y, b, \theta) \\
& \propto \prod_{i=1}^{N}\left[\left(\prod_{j=1}^{n_{i}} \sigma_{i j}^{2}\right)^{-\frac{1}{2}} \exp \left\{-\frac{1}{2}\left(y_{i}-X_{i} \beta-T_{i}^{-1} C_{i} b_{i}\right)^{T}\left(T_{i}^{T} L_{i}^{-T} D_{i}^{-1} L_{i}^{-1} T_{i}\right)\left(y_{i}-X_{i} \beta-T_{i}^{-1} C_{i} b_{i}\right)\right\}\left|G_{b}\right|^{-\frac{1}{2}}\right. \\
& \left.\quad \exp \left(-\frac{1}{2} b_{i}^{T} G_{b}^{-1} b_{i}\right)\right] \times \exp \left(-\frac{1}{2 \sigma_{\beta}^{2}} \beta^{T} \beta\right) \exp \left(-\frac{1}{2 \sigma_{\alpha}^{2}} \alpha^{T} \alpha\right) \exp \left(-\frac{1}{2 \sigma_{\gamma}^{2}} \gamma^{T} \gamma\right) \exp \left(-\frac{1}{2 \sigma_{\lambda}^{2}} \lambda^{T} \lambda\right)\left|G_{b}\right|^{-\frac{v+q+1}{2}} \\
& \quad \exp \left\{-\frac{1}{2} \operatorname{tr}\left(G_{0} G_{b}^{-1}\right)\right\} .
\end{aligned}
$$

To sample from the posterior distribution of the parameters, we develop a simple Gibbs sampler. Full conditional distributions of all parameters have known forms, either normals or inverse Wishart. The full conditional posterior distributions are given below:

- For $b_{i}$

$$
b_{i} \mid y, \theta \sim N\left(\left(C_{i}^{T} L_{i}^{-T} D_{i}^{-1} L_{i}^{-1} C_{i}+G_{b}\right)^{-1} C_{i}^{T} L_{i}^{-T} D_{i}^{-T} D_{i}^{-1} L_{i}^{-1} T_{i}\left(y_{i}-X_{i} \beta\right),\left(C_{i}^{T} L_{i}^{-T} D_{i}^{-1} L_{i}^{-1} C_{i}+G_{b}\right)^{-1}\right) .
$$

- $\operatorname{For} \beta$

$$
\begin{aligned}
\beta \mid y, b, \theta_{-\beta} \sim N & \left(\left(\sum_{i=1}^{N} X_{i}^{T}\left(T_{i}^{T} L_{i}^{-T} D_{i}^{-1} L_{i}^{-1} T_{i}\right) X_{i}+\sigma_{\beta}^{-2} I\right)^{-1}\left(\sum_{i=1}^{N} X_{i}^{T}\left(T_{i}^{T} L_{i}^{-T} D_{i}^{-1} L_{i}^{-1} T_{i}\right)\left(Y_{i}-T_{i}^{-1} C_{i} b_{i}\right)\right),\right. \\
& \left.\left(\sum_{i=1}^{N} X_{i}^{T}\left(T_{i}^{T} L_{i}^{-T} D_{i}^{-1} L_{i}^{-1} T_{i}\right) X_{i}+\sigma_{\beta}^{-2} I\right)^{-1}\right)
\end{aligned}
$$


- For $\alpha$

$$
\begin{aligned}
\alpha \mid y, b, \theta_{-\alpha} \sim N & \left(\left(\sum_{i=1}^{N} W_{i}^{T} L_{i}^{-T} D_{i}^{-1} L_{i}^{-1} W_{i}+\sigma_{\alpha}^{-2} I\right)^{-1}\left(\sum_{i=1}^{N} W_{i}^{T} L_{i}^{-T} D_{i}^{-1} L_{i}^{-1}\left(\left(y_{i}-X_{i} \beta\right)-C_{i} b_{i}\right)\right),\right. \\
& \left.\left(\sum_{i=1}^{N} W_{i}^{T} L_{i}^{-T} D_{i}^{-1} L_{i}^{-1} W_{i}+\sigma_{\alpha}^{-2} I\right)^{-1}\right) .
\end{aligned}
$$

- For $G_{b}$

$$
G_{b} \mid y, b, \theta_{-G_{b}} \sim \operatorname{InvWishart}\left(\left(\sum_{i=1}^{N} b_{i} b_{i}^{T}+G_{0}\right)^{-1}, N+v\right) .
$$

- For $\gamma$ we have the following full conditional:

$$
\begin{aligned}
& p\left(\gamma \mid y, b, \theta_{-\gamma}\right) \\
& \propto \exp \left\{-\frac{1}{2} \sum_{i=1}^{N}\left(y_{i}-X_{i} \beta-T_{i}^{-1} C_{i} b_{i}\right)^{T} T_{i}^{T} L_{i}^{-T} D_{i}^{-1} L_{i}^{-1} T_{i}\left(y_{i}-X_{i} \beta-T_{i}^{-1} C_{i} b_{i}\right)\right\} \exp \left(-\frac{1}{2 \sigma_{\gamma}^{2}} \gamma^{T} \gamma\right) .
\end{aligned}
$$

- For $\lambda$ we have the following full conditional:

$$
\begin{aligned}
& p\left(\lambda \mid y, b, \theta_{-\lambda}\right) \\
& \propto\left(\prod_{i=1}^{N} \prod_{j=1}^{n_{i}} \sigma_{i j}^{2}\right)^{-\frac{1}{2}} \exp \left\{-\frac{1}{2} \sum_{i=1}^{N}\left(y_{i}-X_{i} \beta-T_{i}^{-1} C_{i} b_{i}\right)^{T} T_{i}^{T} L_{i}^{-T} D_{i}^{-1} L_{i}^{-1} T_{i}\left(y_{i}-X_{i} \beta-T_{i}^{-1} C_{i} b_{i}\right)\right\} \\
& \quad \times \exp \left\{-\frac{1}{2 \sigma_{\lambda}^{2}} \lambda^{T} \lambda\right\} .
\end{aligned}
$$

Since the full conditionals (2.12) and (2.13) are not closed, we use a random walk Metropolis-Hastings algorithm, using a normal approximation to the full conditional distribution of $\gamma$ and $\lambda$ as the candidate distribution.

\subsection{Model selection}

To select the best models in Bayesian modeling, there are two typical model selection criteria, posterior predictive loss (PPL) (Gelfand and Ghosh, 1998) and deviance information criterion (DIC) (Spiegelhalter et al., 2002). Both account for goodness of fit and the complexity penalty of model overfitting (Daniels and Hogan, 2008). The PPL quantifies the fit of the model by comparing features of the posterior predictive distribution to equivalent features of the observed data. The comparison is based on a loss function. The DIC is a model-based criterion composed of a goodness of fit term and a penalty term, which is similar to Akaike information criterion (AIC). In this paper, we use DIC for model selection.

Let $\operatorname{Dev}(\theta)=-2 \log L(\theta \mid y)$ be the deviance for $\theta=\left(\beta, \alpha, \gamma, \lambda, G_{b}\right)$ where

$$
L(\theta \mid y)=\prod_{i=1}^{N}(2 \pi)^{-\frac{n_{i}}{2}}\left|\Sigma_{i}\right|^{-\frac{1}{2}} \exp \left\{-\frac{1}{2}\left(y_{i}-X_{i} \beta\right)^{T} \Sigma_{i}^{-1}\left(y_{i}-X_{i} \beta\right)\right\} .
$$




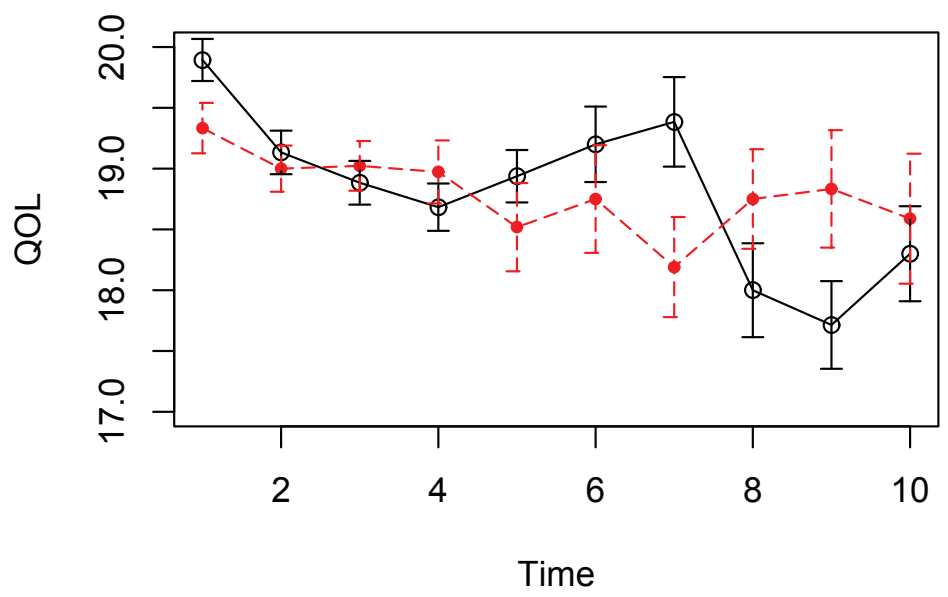

Figure 1: Plot of sample means for two arms (GEFITINIB (Solid line) and ERLOTINIB (dashed line)).

Then the DIC is defined as

$$
\begin{aligned}
\mathrm{DIC} & =2 \overline{\operatorname{Dev}(\theta)}-\operatorname{Dev}(\bar{\theta}) \\
& =\overline{\operatorname{Dev}(\theta)}+p_{D},
\end{aligned}
$$

where $\overline{\operatorname{Dev}(\theta)}$ is the posterior mean deviance, $\operatorname{Dev}(\bar{\theta})=\operatorname{Dev}\left[E_{\theta}(\theta \mid y)\right]$, and $p_{D}=\overline{\operatorname{Dev}(\theta)}-\operatorname{Dev}(\bar{\theta})$. Note that the first term in (2.14) measures the fit by the deviance and the second term, which is called penalty term, measures model complexity and $p_{D}$ is the effective number of parameters.

\section{Lung cancer study}

\subsection{Data description}

Kim et al. (2012) reported data from a 92-week longitudinal study in 95 patients with lung cancer. The study was a prospective open-label randomized non-comparative parallel study in a single institution (Kim et al., 2012). The study evaluated the response rate for two treatment arms (GEFITINIB or ERLOTINIB). The response rate means the percentage of patients whose cancer shrinks or disappears after treatment. It was of interest to see if there was a negative impact of treatment on a patient's quality of life (QOL) which was analyzed in Lee et al. (2016).

We focused on the European Organization for Research and Treatment of Cancer Quality of Life Questionnaire Lung Cancer (EORTC QLQ-LC13) as a standard instrument to assess the quality of life for lung cancer patients. Patients completed the questionnaire before receiving the first treatment dose at baseline, on day 1 of each subsequent 29-day cycle (4 weeks), and at the end of the study (92 weeks). Since maximum number of cycles was 23 , we need a model that 0 captures a long serial correlation structure of the covariance matrix. Each questionnaire was originally scored on a 4-point scale ranging from normal, 'Not at all' (1) to abnormal, 'Very much' (4). Lee et al. (2016) focused on sum of the 12-item questionnaire module (SQOL) that revealed no significant difference between two treatments arms. Figure 1 presents sample means and 95\% confidence intervals of the means for first 40 weeks (first 10 months). The sample means for two arms were statistically different at baseline. Then there was no difference between two arm until the fifth visit. However, there was significantly different after the fifth visit. In this paper, we consider first 40 weeks (first 10 months) and we analyze 
Table 1: Models for $\phi_{i j l}, l_{i j k}$, and $\log \sigma_{i j}^{2}$ in the lung cancer example

\begin{tabular}{|c|c|c|c|}
\hline Model & GARP & GMAP & $\log (\mathrm{IV})$ \\
\hline AR(1)-1 & $\phi_{i j k}=\alpha_{0} I_{(|j-k|=1)}$ & & $\log \sigma_{i j}^{2}=\lambda_{0}+\lambda_{1} \operatorname{Arm}_{i}$ \\
\hline $\mathrm{AR}(1)-2$ & $\phi_{i j k}=\alpha_{0} I_{(|j-k|=1)}$ & & $\log \sigma_{i j}^{2}=\lambda_{0}+\lambda_{1} t_{i j}$ \\
\hline $\operatorname{AR}(1)-3$ & $\phi_{i j k}=\alpha_{0} I_{(|j-k|=1)}$ & & $\log \sigma_{i j}^{2}=\lambda_{0}+\lambda_{1} \operatorname{Arm}_{i}+\lambda_{2} t_{i j}$ \\
\hline $\operatorname{AR}(2)-1$ & $\phi_{i j k}=\alpha_{0} I_{(|j-k|=1)}+\alpha_{1} I_{(|j-k|=2)}$ & & $\log \sigma_{i j}^{2}=\lambda_{0}+\lambda_{1} \operatorname{Arm}_{i}$ \\
\hline $\operatorname{AR}(2)-2$ & $\phi_{i j k}=\alpha_{0} I_{(|j-k|=1)}+\alpha_{1} I_{(|j-k|=2)}$ & & $\log \sigma_{i j}^{2}=\lambda_{0}+\lambda_{1} t_{i j}$ \\
\hline $\operatorname{AR}(2)-3$ & $\phi_{i j k}=\alpha_{0} I_{(|j-k|=1)}+\alpha_{1} I_{(|j-k|=2)}$ & & $\log \sigma_{i j}^{2}=\lambda_{0}+\lambda_{1} \operatorname{Arm}_{i}+\lambda_{2} t_{i j}$ \\
\hline MA(1)-1 & & $l_{i j k}=\gamma_{0} I_{(|j-k|=1)}$ & $\log \sigma_{i j}^{2}=\lambda_{0}+\lambda_{1} \operatorname{Arm}_{i}$ \\
\hline MA(1)-2 & & $l_{i j k}=\gamma_{0} I_{(|j-k|=1)}$ & $\log \sigma_{i j}^{2}=\lambda_{0}+\lambda_{2} t_{i j}$ \\
\hline MA(1)-3 & & $l_{i j k}=\gamma_{0} I_{(|j-k|=1)}$ & $\log \sigma_{i j}^{2}=\lambda_{0}+\lambda_{1} \operatorname{Arm}_{i}+\lambda_{2} t_{i j}$ \\
\hline MA(2)-1 & & $l_{i j k}=\gamma_{0} I_{(|j-k|=1)}+\gamma_{1} I_{(|j-k|=2)}$ & $\log \sigma_{i j}^{2}=\lambda_{0}+\lambda_{1} \operatorname{Arm}_{i}$ \\
\hline MA(2)-2 & & $l_{i j k}=\gamma_{0} I_{(|j-k|=1)}+\gamma_{1} I_{(|j-k|=2)}$ & $\log \sigma_{i j}^{2}=\lambda_{0}+\lambda_{2} t_{i j}$ \\
\hline MA(2)-3 & & $l_{i j k}=\gamma_{0} I_{(|j-k|=1)}+\gamma_{1} I_{(|j-k|=2)}$ & $\log \sigma_{i j}^{2}=\lambda_{0}+\lambda_{1} \operatorname{Arm}_{i}+\lambda_{2} t_{i j}$ \\
\hline $\operatorname{ARMA}(1,1)-1$ & $\phi_{i j k}=\alpha_{0} I_{(|j-k|=1)}$ & $l_{i j k}=\gamma_{0} I_{(|j-k|=1)}$ & $\log \sigma_{i j}^{2}=\lambda_{0}+\lambda_{1} \operatorname{Arm}_{i}$ \\
\hline $\operatorname{ARMA}(1,1)-2$ & $\phi_{i j k}=\alpha_{0} I_{(|j-k|=1)}$ & $l_{i j k}=\gamma_{0} I_{(|j-k|=1)}$ & $\log \sigma_{i j}^{2}=\lambda_{0}+\lambda_{2} t_{i j}$ \\
\hline $\operatorname{ARMA}(1,1)-3$ & $\phi_{i j k}=\alpha_{0} I_{(|j-k|=1)}$ & $l_{i j k}=\gamma_{0} I_{(|j-k|=1)}$ & $\log \sigma_{i j}^{2}=\lambda_{0}+\lambda_{1} \operatorname{Arm}_{i}+\lambda_{2} t_{i j}$ \\
\hline
\end{tabular}

GARP $=$ generalized autoregressive parameter; GMAP $=$ generalized moving average parameter; IV = innovation variance.

SQOL data using our proposed model. The main question of interest in this analysis was: Is there any improvement of patients' quality of life between GEFITINIB and ERLOTINIB arms?

To examine treatment differences in SQOL, we included type of treatment (ARM $=1$ for GEFITINIB, 0 for ERLOTINIB) and week number $(0,4,8, \ldots, 92)$; the latter was re-scaled (TIME = $0.0,0.4,0.8, \ldots, 9.2)$. We assumed the missing responses were missing at random as in Lee $e$ al. (2016).

\subsection{Models fit}

We considered models with random intercept and various structures of $\Sigma_{i}$ which included ARM and visit time $t_{i j}$. In particular, we considered the models specified in Table 1.

We fit nine models for $\Sigma_{i}$ using various structures specified in Table 1. AR(2)-1 and MA(2)1 indicates models with a heteroscedastic covariance matrix with $\operatorname{AR}(2)$ and $M A(2)$ structure and IV depending on arm, respectively. AR(2)-2 and MA(2)-2 indicate models with a heteroscedastic covariance matrix with $\mathrm{AR}(2)$ and $\mathrm{MA}(2)$ structure and IV linear in time, respectively. $\mathrm{AR}(2)-3$ and MA(2)-3 indicate models with a heteroscedastic covariance matrix with $\operatorname{AR}(2)$ and MA(2) structure and IV linear in both arm and time, respectively. $\operatorname{ARMA}(1,1)-1,2$, and 3 indicate a model with a heteroscedastic covariance matrix with an $\operatorname{ARMA}(1,1)$ structure and IV depending on ARM, linearity of time, and both.

As the simple random intercept form of models is often adequate, we only discuss random intercept models, $c_{i j}^{T} b_{i}=b_{i 0} \sim N\left(0, G_{b}\right)$ in $(2.1)$, and prior for $G_{b}$ is an inverse gamma $(0.1,0.1)$. In practice, hyperparameters in (2.6)-(2.9) are $\sigma_{\beta}^{2}=\sigma_{\alpha}^{2}=\sigma_{\gamma}^{2}=100$ and $\sigma_{\lambda}^{2}=10$.

The convergence of the Gibbs sampler was monitored by examining time series plots of the parameters over iteration and the Gelman and Rubin approach of using multiple chains (Gelman and Rubin, 1992). For all models considered, convergence to the posterior distribution was reached at about 1,000 iterations and the mixing was good. In practice, we used sample of 10,000 after burn-in 
Table 2: DICs and $P_{D}$ s for the models considered

\begin{tabular}{crrrrrrrrr}
\hline \hline Model & AR(1)-1 & AR(1)-2 & AR(1)-3 & AR(2)-1 & AR(2)-2 & AR(2)-3 & MA(1)-1 & MA(1)-2 & MA(1)-3 \\
\hline DIC & 4149.651 & 4141.687 & 4141.491 & 4115.231 & 4103.471 & 4102.764 & 4493.138 & 4515.747 & 4496.947 \\
$p_{D}$ & 7.629 & 7.501 & 8.498 & 8.293 & 8.222 & 9.122 & 156.596 & 168.455 & 161.253 \\
\hline \multirow{2}{*}{ Model } & \multirow{2}{*}{ MA(2)-1 } & \multirow{2}{*}{ MA(2)-2 } & \multirow{2}{*}{ MA(2)-3 } & ARMA & ARMA & ARMA & & & \\
& & $(1,1)-1$ & $(1,1)-2$ & $(1,1)-3$ & & & \\
\hline DIC & 4377.705 & 4390.108 & 4395.659 & 4116.265 & 4104.163 & 4102.862 & & & \\
$p_{D}$ & 125.888 & 132.471 & 134.057 & 8.149 & 8.266 & 8.969 & & & \\
\hline \hline
\end{tabular}

DIC $=$ deviance information criterion; $p_{D}=$ effective number of parameters.

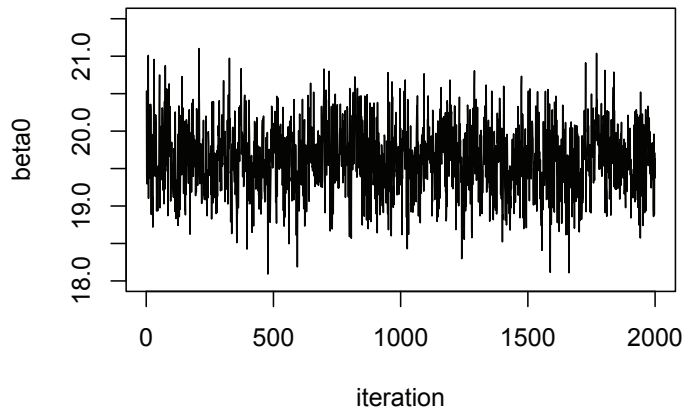

(a) $\beta_{0}$

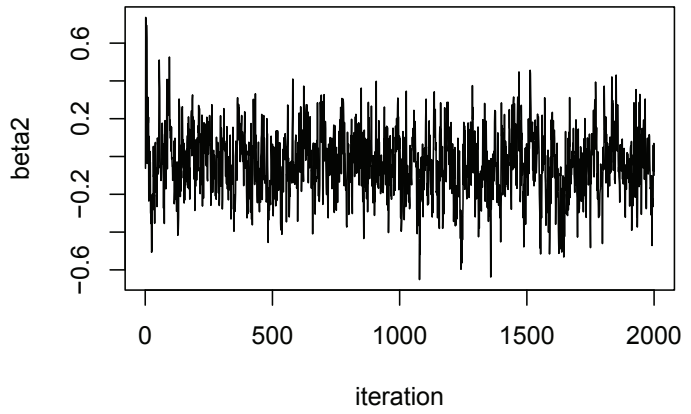

(c) $\beta_{2}$

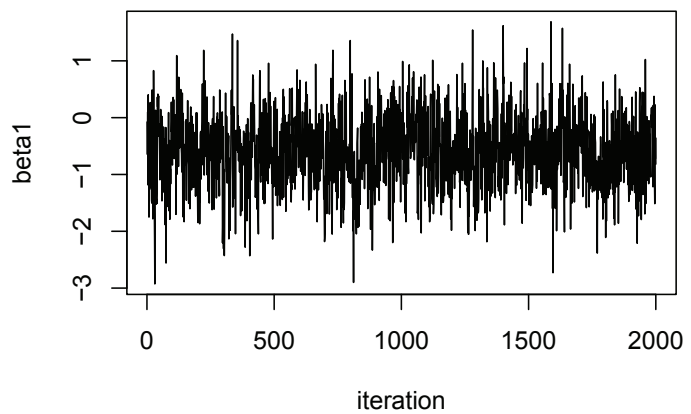

(b) $\beta_{1}$

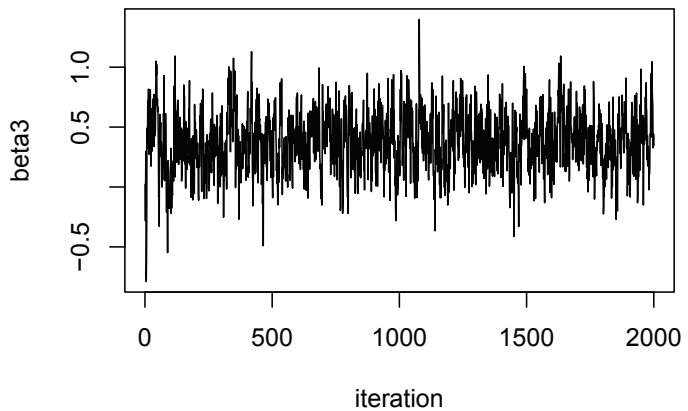

(d) $\beta_{3}$

Figure 2: Convergence check of estimators for model ARMA(1,1)-3.

period of 5,000.

We used the DIC given by (2.14) to compare the models. Comparison of DICs are presented in Table 2. The DIC values for $\operatorname{ARMA}(1,1)$ were dominant over those for $\operatorname{AR}(1)$ or MA(1) which are commonly used in longitudinal data analysis. The DIC values for models with $\operatorname{ARMA}(1,1)$ were comparable to those for models with $\mathrm{AR}(2)$. It indicates that practitioners often consider ARMA models when a high-order AR structure of the covariance matrix is required to capture the dependence structure (Judge et al., 1980). In our case, the maximum replication numbers was 10 and is not very large. Therefore, the DIC values for the model $\operatorname{AR}(2)-2, \operatorname{AR}(2)-3, \operatorname{ARMA}(1,1)-2$, and $\operatorname{ARMA}(1,1)-3$ were comparable.

The effective number of parameters, $p_{D}$, was roughly what we would expect for the models considered. In the four models, the effective numbers in the other models were less than the expected ones. This general phenomenon is observed with the DIC (Spiegelhalter et al., 2002). 
Table 3: Maximum likelihood estimates for models AR(2)-2, AR(2)-3, ARMA(1,1)-2, and ARMA(1, 1)-3 fit the lung cancer data

\begin{tabular}{ccccc}
\hline \hline & AR(2)-2 & AR(2)-3 & ARMA $(1,1)-2$ & ARMA(1, 1$)-3$ \\
\hline$\beta_{0}$ (Int.) & $19.644^{*}$ & $19.650^{*}$ & $19.613^{*}$ & $19.624^{*}$ \\
& $(18.734,20.555)$ & $(18.778,20.522)$ & $(18.728,20.497)$ & $(18.782,20.465)$ \\
$\beta_{1}$ (Arm) & -0.601 & -0.604 & -0.575 & -0.620 \\
& $(-1.894,0.692)$ & $(-1.879,0.672)$ & $(-1.816,0.666)$ & $(-1.834,0.594)$ \\
$\beta_{2}$ (Time) & -0.058 & -0.061 & -0.033 & -0.024 \\
& $(-0.392,0.275)$ & $(-0.379,0.257)$ & $(-0.360,0.294)$ & $(-0.324,0.277)$ \\
$\beta_{3}$ (Arm*Time) & 0.342 & 0.345 & 0.376 & 0.355 \\
& $(-0.157,0.841)$ & $(-0.144,0.834)$ & $(-0.102,0.854)$ & $(-0.113,0.822)$ \\
\hline$\alpha_{0}$ & $0.458^{*}$ & $0.453^{*}$ & $0.764^{*}$ & $0.764^{*}$ \\
& $(0.383,0.532)$ & $(0.379,0.527)$ & $(0.699,0.828)$ & $(0.702,0.826)$ \\
$\alpha_{1}$ & $0.259^{*}$ & $0.261^{*}$ & & \\
& $(0.182,0.337)$ & $(0.183,0.339)$ & & $-0.372^{*}$ \\
$\gamma_{0}$ & & & $-0.361^{*}$ & $(-0.479,-0.264)$ \\
& & & $(-0.469,-0.254)$ & $2.308^{*}$ \\
$\lambda_{0}$ & $2.440^{*}$ & $2.350^{*}$ & $2.399^{*}$ & $(2.122,2.495)$ \\
& $(2.288,2.592)$ & $(2.164,2.536)$ & $(2.243,2.554)$ & 0.169 \\
$\lambda_{1}$ & & $-0.094^{*}$ & & $(-0.039,0.376)$ \\
& & $(-0.143,-0.045)$ & -0.097 \\
$\lambda_{2}$ & $-0.100^{*}$ & 0.167 & $-0.099^{*}$ & $(-0.147,0.048)$ \\
\hline$G_{b}$ & $(-0.146,-0.051)$ & $(-0.042,0.377)$ & $(-0.148,-0.051)$ & $1.045^{*}$ \\
& $1.077^{*}$ & $1.078^{*}$ & $1.048^{*}$ & $(0.554,1.537)$ \\
\hline DIC & $(0.444,1.709)$ & $(0.437,1.720)$ & $(0.540,1.556)$ & 4102.862 \\
$P_{d}$ & 4103.471 & 4102.764 & 4104.163 & 8.969 \\
\hline \hline Parameter estimates with standard errors in the parentheses. ${ }^{*}$ indicates the $95 \%$ credible interval does not include zero.
\end{tabular}

To examine the convergence of Markov chains, Figure 2 shows trace plots of $\beta$ for the model ARMA(1, 1)-3. The figure shows that Markov chains converge to the target distribution. Therefore, we concluded that the Markov chains converged properly for the estimation of our model.

\subsection{Results}

Table 3 presents posterior means and 95\% credible intervals for the four models considered. Posterior means of all coefficients of covariates were similar for the four models. We focus on models AR(2)-3 and ARMA(1, 1)-3 which had the smallest DIC. In model AR(2)-3, the 95\% credible interval for the intercept $\left(\lambda_{0}\right)$ in the $\log (\mathrm{IV})$ was above zero and that for the coefficient of Arm $\left(\lambda_{1}\right)$ was below zero. The interval for the coefficient of Time $\left(\lambda_{2}\right)$ included zero. The results indicate that random variation for GEFITINIB arm was smaller than that for ERLOTINIB arm. Therefore, the LMM was explicitly heterogeneous within subject variances. The posterior mean of GARPs $\left(\alpha_{0}, \alpha_{1}\right)$ for coefficients of $\operatorname{AR}(1)$ and $\operatorname{AR}(2)$ were positive; therefore, there was $\operatorname{AR}(2)$ structure for the within-subject variation. The posterior mean of $G_{b}$ indicates between-subject variance. The $95 \%$ credible intervals of coefficients for all covariates included zero; therefore, there were no effects for all covariates on the conditional mean of the quality of life for lung cancer patients.

In model ARMA $(1,1)-3$, the $95 \%$ credible interval for the intercept $\left(\lambda_{0}\right)$ in the $\log (\mathrm{IV})$ was above zero and those for Arm $\left(\lambda_{1}\right)$ and Time $\left(\lambda_{2}\right)$ included zero. The interval for GARPs $\left(\alpha_{0}\right)$ and GMAP $\left(\gamma_{0}\right)$ were above zero and below zero, respectively. The $95 \%$ credible intervals of coefficients for all covariates included zero; therefore, there were no effects of all covariates on the conditional mean of the quality of life. 


\section{Conclusion}

We have proposed linear mixed models with a heterogeneous covariance matrix with ARMA structure. The covariance matrix is modeled via the ARMA modified Cholesky decomposition that represents a computationally attractive approach. The covariance matrix is factored into GARPs, GMAPs, and IVs. The GARPs and GMAPs explain the ARMA serial correlation of repeated outcomes. The marginal covariance matrix is positive definite when both random effects covariance matrix is positive definite and the IVs are all positive. In addition, the approach provides better forecasting performance than competing higher order AR or MA models.

The proposed models were fitted using Bayesian approach. Gibbs sampler with the random walk Metropolis-Hastings algorithm was used for parameter estimation. DIC was used to select the best models in Bayesian modeling.

The parameters of the covariance matrix with the ARMA and AR structure were better than those with MA structure for the analysis of lung cancer data. The $95 \%$ credible intervals of coefficients of covariates included zeros; therefore, the patients' SQOL were the same between two arms.

Currently, there is further ongoing research on possibilities for extensions to generalized linear mixed models.

\section{Acknowledgement}

This work was supported by Basic Science Research Program through the National Research Foundation of Korea (KRF) funded by the Ministry of Education, Science and Technology (NRF-2014R1A1A 2054997, NRF-2016R1D1A1B03930343). We would like to thank Drs. Myung-Ju Ahn and Insuk Sohn of Samsung Medical Center in South Korea for providing the data and for their help in data collection and clarifying some issues with data.

\section{References}

Daniels MJ and Zhao YD (2003). Modelling the random effects covariance matrix in longitudinal data, Statistics in Medicine, 22, 1631-1647.

Daniels MJ and Hogan JW (2008). Missing Data in Longitudinal Studies: Strategies for Bayesian Modeling and Sensitivity Analysis, Chapman \& Hall/CRC, Boca Raton, FL.

Diggle PJ, Heagerty P, Liang KY, and Zeger SL (2002). Analysis of Longitudinal Data (2nd ed), Oxford University Press, Oxford.

Gelfand AE and Ghosh SK (1998). Model choice: a minimum posterior predictive loss approach, Biometrika, 85, 1-11.

Gelman A and Rubin DB (1992). Inference from iterative simulation using multiple sequences, Statistical Science, 7, 457-511.

Hansen PR and Lunde A (2005). A forecast comparison of volatility models: does anything beat a GARCH $(1,1)$, Journal of Applied Econometrics, 20, 873-889.

Judge GG, Griffiths WE, Hill RC, and Lee TC (1980). The Theory and Practice of Ecomometrics, Wiley, New York.

Kim J, Sohn I, and Lee K (2016). Bayesian modeling of random effects precision/covariance matrix in cumulative logit random effects models, Working paper.

Kim ST, Uhm JE, Lee J, Sun J, Sohn I, Kim SW, Jung SH, Park YH, Ahn JS, Park K, and Ahn MJ (2012). Randomized phase II study of gefitinib versus erlotinib in patients with advanced non-small cell lung cancer who failed previous chemotherapy, Lung Cancer, 75, 82-88. 
Laird NM and Ware JH (1982). Random-effects models for longitudinal data, Biometrics, 38, 963974.

Lee K (2013). Bayesian modeling of random effects covariance matrix for generalized linear mixed models, Communications for Statistical Applications and Methods, 20, 235-240.

Lee K, Baek C, and Daniels MJ (2016). ARMA Cholesky factor models for longitudinal regression models, Working paper.

Lee K, Lee J, Hagan J, and Yoo JK (2012). Modeling the random effects covariance matrix for the generalized linear mixed models, Computational Statistics \& Data Analysis, 56, 1545-1551.

Lee K and Sung S (2014). Autoregressive Cholesky factor modeling for marginalized random effects models, Communications for Statistical Applications and Methods, 21, 169-181.

Lee K and Yoo JK (2014). Bayesian Cholesky factor models in random effects covariance matrix for generalized linear mixed models, Computational Statistics \& Data Analysis, 80, 111-116.

Pan J and MacKenzie G (2003). On modelling mean-covariance structures in longitudinal studies, Biometrika, 90, 239-244.

Pan J and MacKenzie G (2006). Regression models for covariance structures in longitudinal studies, Statistical Modelling, 6, 43-57.

Pan J and MacKenzie G (2007). Modelling conditional covariance in the linear mixed model, Statistical Modelling, 7, 49-71.

Pourahmadi M (1999). Joint mean-covariance models with applications to longitudinal data: unconstrained parameterisation, Biometrika, 86, 677-690.

Pourahmadi M (2000). Maximum likelihood estimation of generalised linear models for multivariate normal covariance matrix, Biometrika, 87, 425-435.

Spiegelhalter DJ, Best NG, Carlin BP, and van der Linde A (2002). Bayesian measures of model complexity and fit, Journal of the Royal Statistical Society Series B (Statistical Methodology), 64, 583-639.

Zhang W and Leng C (2012). A moving average Cholesky factor model in covariance modelling for longitudinal data, Biometrika, 99, 141-150. 